\title{
Clinical and Microbiological Profile of Urinary Tract Infections in Diabetic versus Non-Diabetic Individuals
}

\author{
Ravi Kumar ${ }^{1}$, Rajesh Kumar ${ }^{2}$, Prinka Perswani ${ }^{2}$, Muhammad Taimur ${ }^{3}$, Ali Shah ${ }^{1}$, Faizan Shaukat ${ }^{4}$ \\ 1. Internal Medicine, Jinnah Sindh Medical University, Karachi, PAK 2. Internal Medicine, Liaquat University of Medical \\ and Health Sciences, Hyderabad, PAK 3. Internal Medicine, Dow University of Health Sciences, Karachi, PAK 4. Internal \\ Medicine, Jinnah Postgraduate Medical Center, Karachi, PAK
}

Corresponding author: Faizan Shaukat, faizan.shaukat89@gmail.com

\section{Abstract}

\section{Introduction}

Diabetic patients have a higher tendency of developing all infections, especially infections of the genitourinary tract. In most cases, urinary tract infections (UTI) in diabetic patients are asymptomatic. The aim of this study to was to compare the incidence and clinical and microbiological features of UTI between diabetic and non-diabetic patients.

\section{Methods}

In this prospective, comparative study, the incidence and clinical and microbiological features of UTI were compared between diabetic and non-diabetic patients via consecutive non-probability sampling technique. For every diabetic patient, one non-diabetic control was included. All patients were screened for UTI through a midstream urinary sample. Their demographic characteristics, clinical profile, and urinary microscopy were compared. Data were entered and analyzed using SPSS version 22.0.

\section{Results}

In the diabetes group, 35/256 (13.67\%) patients had culture-positive UTI as compared to 18/250 (7.2\%) in the non-diabetic group. Diabetic group had twice the risk of UTI ( $p=0.01$; odds ratio [OR]: 2.04; confidence interval [CI]: $1.12,3.71)$ and female gender in the diabetic group had a risk of almost five times $(p=0.01$; OR: 4.93; CI: $1.12,20.16$ ) that of the non-diabetic group. In the diabetic group, $31.4 \%$ patients were asymptomatic as compared to $5.6 \%$ in the non-diabetic group ( $p=0.03$; OR: 7.79 ; CI: $0.92,66.18)$. E. coli was the most commonly identified microorganism in both groups. Pseudomonas aeruginosa was identified in $14 \%$ of diabetic cases and none in the non-diabetic.

Received 08/14/2019

Review began 08/16/2019 Review ended 08/18/2019 Published 08/22/2019

\section{(c) Copyright 2019}

Kumar et al. This is an open access article distributed under the terms of the Creative Commons Attribution License CC-BY 3.0., which permits unrestricted use, distribution, and reproduction in any medium, provided the original author and source are credited.

\section{Conclusions}

UTIs are more frequent among diabetics. Asymptomatic bacteriuria is a more common entity in diabetic patients and does not require any treatment.

Categories: Endocrinology/Diabetes/Metabolism, Internal Medicine, Infectious Disease

Keywords: urinary tract infections, diabetic vs non-diabetic, escherichia coli (e. coli), pseudomonas aeruginosa, pakistan

\section{Introduction}

Diabetes mellitus is associated with immune system dysfunction, which makes the affected individuals prone to repeated infections, especially infections of the genitourinary tract. Diabetic patients have more than twice the tendency of developing genitourinary tract infections [1]. The altered host responses in diabetic patients cause increased adherence of the microorganisms to the uroepithelial cells, granulocyte dysfunction, and altered intracellular calcium metabolism. This results in increased susceptibility of these individuals to develop urinary tract infections (UTI) [2-3]. Another important pathology underlying the increased incidence of UTIs and recurrent UTIs in the diabetic population is diabetic bladder dysfunction. It affects more than half of the people with longstanding and poorly controlled diabetes. It includes storage problems as well as voiding problems [4]. Furthermore, some pathogens flourish well in high glucose environment [5].

UTIs may be symptomatic or asymptomatic [6]. The common symptoms include burning micturition, urgency, dysuria, cramping in the lower abdomen, mental irritability, back or flank pain, chill, nausea, fever, vomiting, fatigue, and weakness [7]. Escherichia coli (E. coli) is the prominent causative pathogen of UTI in both diabetic and non-diabetic populations, followed by coagulase-negative Staphylococci (CONs), Enterococcus species (spp.), Candida albicans, and non-albicans Candida spp. [6,8]. It is very important to 
screen diabetic patients for UTI for timely diagnosis, complete treatment, and prevention of progression to renal complications and ultimately severe renal failure. However, there are controversies regarding the incidence, clinical pattern, and microbiology of UTIs in diabetic individuals as compared to those in nondiabetic ones [5]. Hence, this study was conducted to compare the incidence and clinical and microbiological features of UTIs between diabetic and non-diabetic patients. It would help assess the frequency of UTIs in the diabetic population and enable the diabetologists predict the clinical and microbiological patterns of UTI in their patients.

\section{Materials And Methods}

This prospective, observational, cross-sectional, comparative study was conducted from July 1, 2017 to February 28, 2018 in the outpatient department (OPD) of a general hospital in Pakistan. Ethical approval from the institute was acquired. Consecutive non-probability sampling technique was adapted. All patients of type II diabetes mellitus, of both genders and age 18 years and above, were recruited after informed consent. All patients were screened for UTI through a midstream 5-ml urinary sample. The presence of bacteria, positive leukocyte esterase, and white blood count $(\mathrm{WBC})>5$ per high power field (HPF) were taken as diagnostic for UTI. Pyuria was defined as WBC $>10 / \mathrm{HPF}$, and hematuria was defined as red blood cells $>5 / \mathrm{HPF}$. Urinary culture analysis, for identification of the pathogen, was performed only for patients who were found to be infective on urine microscopy.

Clinical and demographic characteristics included in the study were as follows: age, gender, and HbA1c for both groups. For the diabetic group, duration of diabetes, diabetes-related complications, and the presence of diabetic kidney disease were also noted. Patients who had taken antibiotics within the last two weeks for any reason were not included in this study. Patients with anatomical and neurologic urinary tract abnormalities, pregnant women, cases of complicated UTI (including pylonephritis), and patients with acute and/or chronic renal failure were also excluded.

During the study period, 260 diabetic patients were recruited. For every diabetic patient, a non-diabetic patient was included. The non-diabetic control group was selected from the attendants of the diabetic group to align their sociodemographic characteristics. By the end of the study, there were 256 records in the diabetes group and 250 records in the non-diabetes group. Their data was managed using SPSS for Windows version 22.0 (IBM Corp., Armonk, NY). The mean and standard deviation were calculated for continuous variables such as age, duration of diabetes, and $\mathrm{HbA1c}$ levels. Frequency and percentages were calculated for categorical variables. The incidence of UTI was compared for the diabetic and non-diabetic groups. Chisquare was applied for comparison. $P$-value $\leqslant 0.05$ was taken as significant. Odds ratio $(\mathrm{OR})$ and confidence interval (CI) were calculated.

\section{Results}

Among 256 patients in the diabetic group, there were 112 (43.7\%) males and 144 (56.3\%) females. Their mean age was $56 \pm 11$ years. Non-diabetic patients were relatively younger with a mean age of $48 \pm 12$ years. There were more women $(n=156 ; 62.4 \%)$ than men $(n=94 ; 37.6 \%)$ in the non-diabetic group. Demographic and clinical characteristics of both study groups are compared in Table 1 . 


\section{Cureus}

\begin{tabular}{|c|c|c|}
\hline Patient Characteristics & Diabetic Group $(n=256)$ & Non-diabetic Group $(n=250)$ \\
\hline \multicolumn{3}{|l|}{ Gender } \\
\hline Male & $112(43.7 \%)$ & $94(37.6 \%)$ \\
\hline Female & $144(56.3 \%)$ & $156(62.4 \%)$ \\
\hline \multicolumn{3}{|l|}{ Age in years } \\
\hline Mean & $56 \pm 11$ & $48 \pm 12$ \\
\hline Less than 40 years & $38(14.8 \%)$ & $72(28.8 \%)$ \\
\hline 40-60 years & $115(44.9 \%)$ & $87(34.8 \%)$ \\
\hline Above 60 years & $103(40.2 \%)$ & $91(36.4 \%)$ \\
\hline \multicolumn{3}{|c|}{ Duration of diabetes in years } \\
\hline Mean & $7.6 \pm 3.8$ & \multirow{5}{*}{ Not applicable } \\
\hline Less than 5 years & $78(30.5 \%)$ & \\
\hline 5-10 years & $97(37.8 \%)$ & \\
\hline More than 10 years & $81(31.6 \%)$ & \\
\hline \multicolumn{2}{|c|}{ Diabetic complications (any) } & \\
\hline Yes & $118(46.1 \%)$ & \multirow{2}{*}{ Not applicable } \\
\hline No & $138(53.9 \%)$ & \\
\hline \multicolumn{3}{|c|}{ Diabetes-related kidney disease } \\
\hline Yes & $34(13.3 \%)$ & \multirow{3}{*}{ Not applicable } \\
\hline No & $222(86.7 \%)$ & \\
\hline \multicolumn{2}{|c|}{ Glycosylated haemoglobin A1c (\%) } & \\
\hline Mean & $7.8 \pm 2.6$ & $4.9 \pm 1.2$ \\
\hline Less than $7 \%$ & $48(18.7 \%)$ & $250(100 \%)$ \\
\hline $7 \%-8.5 \%$ & $134(52.3 \%)$ & \multirow{2}{*}{ Not applicable } \\
\hline More than $8.5 \%$ & $74(28.9 \%)$ & \\
\hline
\end{tabular}

TABLE 1: Demographic and clinical characteristics of participants in the diabetic group $(n=256)$ and non-diabetic group $(n=250)$

In diabetes group, 35 (13.67\%) patients were identified with culture positive UTI as compared to 18 (7.2\%) participants in non-diabetic group. In both groups, UTI was more common in female gender. Diabetic group had an overall twice risk of UTI ( $p=0.01$; OR: 2.04 ; CI: $1.12,3.71)$ and female gender in diabetic group had a risk of almost five times ( $p=0.01$; OR: 4.93 ; CI: $1.12,20.16$ ) that of the non-diabetic group of developing urinary tract infection (Table 2). 


\section{Cureus}

\begin{tabular}{|c|c|c|c|c|c|}
\hline & Diabetic Group ( $\mathrm{n}=256$ ) & Non-diabetic Group ( $n=250$ ) & P-value & Odds Ratio & Confidence Interval \\
\hline Total & $35(13.7 \%)$ & $18(7.2 \%)$ & 0.01 & 2.04 & $1.12,3.71$ \\
\hline Male & 4/35 (11.4\%) & $7 / 18(38.9 \%)$ & \multirow{2}{*}{0.01} & 0.2 & $0.05,0.83$ \\
\hline Female & $31 / 35(88.6 \%)$ & $11 / 18(61.1 \%)$ & & 4.93 & $1.12,20.16$ \\
\hline
\end{tabular}

TABLE 2: Incidence of urinary tract infection in the diabetic group $(n=256)$ and non-diabetic group $(n=250)$

Almost 30\% patients in the diabetic group with culture-proven UTI were asymptomatic as compared to only $5 \%$ in the non-diabetic group ( $p=0.03$; OR: 7.79; CI: 0.92, 66.18). There was no other significant difference between the presentations of UTI in the two groups, as shown in Table 3 .

\begin{tabular}{|c|c|c|c|c|c|}
\hline Signs / Symptoms & Diabetic Group ( $n=35$ ) & Non-diabetic Group $(n=18)$ & P-value & Odds Ratio & Confidence Interval \\
\hline No signs / symptoms & $11(31.4 \%)$ & $1(5.6 \%)$ & 0.03 & 7.79 & $0.92,66.18$ \\
\hline Fever & $21(60.0 \%)$ & $11(61.1 \%)$ & 0.93 & 0.95 & $0.3,3.06$ \\
\hline Dysuria & $17(48.5 \%)$ & $11(61.1 \%)$ & 0.38 & 0.6 & $0.19,1.91$ \\
\hline Increased frequency ( $\geq 5 /$ day) & $13(37.1 \%)$ & 7 (38.9\%) & 0.90 & 0.93 & $0.29,2.99$ \\
\hline Dribbling & $9(25.7 \%)$ & $5(27.8 \%)$ & 0.87 & 0.9 & $0.25,3.24$ \\
\hline Abdominal / flank pain & $9(25.7 \%)$ & $5(27.8 \%)$ & 0.87 & 0.9 & $0.25,3.24$ \\
\hline Pyuria & $7(20.0 \%)$ & $2(11.1 \%)$ & 0.41 & 2.0 & $0.37,10.81$ \\
\hline Vomiting & $4(11.4 \%)$ & $2(11.1 \%)$ & 0.97 & 1.03 & $0.17,6.25$ \\
\hline Urinary retention & $4(11.4 \%)$ & $2(11.1 \%)$ & 0.97 & 1.03 & $0.17,6.25$ \\
\hline Hematuria & $2(5.7 \%)$ & $1(5.6 \%)$ & 0.98 & 1.03 & $0.09,12.19$ \\
\hline
\end{tabular}

TABLE 3: Clinical and incidence of urinary tract infection in the diabetic group $(n=256)$ and nondiabetic group $(n=250)$

E. coli was the most commonly identified microorganism in both diabetic and non-diabetic groups. $P$. aeruginosa was identified in $14 \%$ of diabetic cases. Other organisms included Klebsiella species and Enterobacter species (Table 4). 


\section{Cureus}

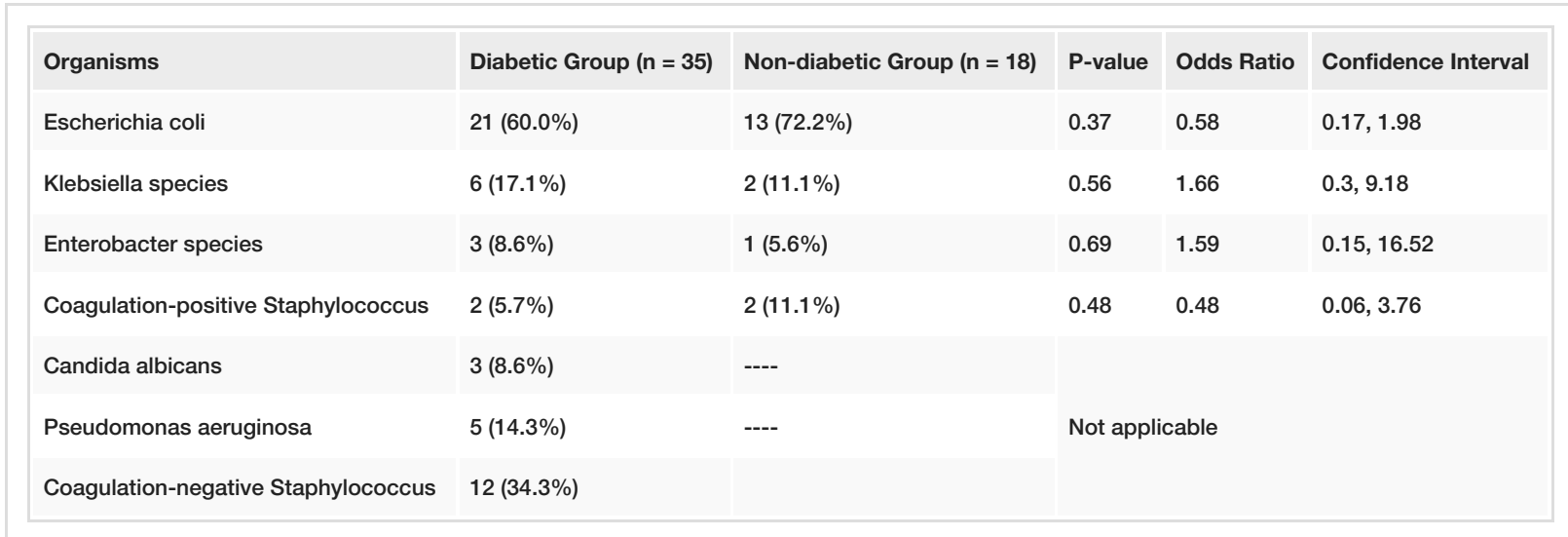

TABLE 4: Microorganisms identified in the diabetic group $(n=35)$ and non-diabetic group $(n=18)$ on urine culture

\section{Discussion}

This study compared the incidence of UTIs in demographically comparable groups of diabetic and nondiabetic individuals. There was an overall significantly higher incidence of UTIs in the diabetic group; these individuals had twice the risk as compared to non-diabetics. Females also showed a significantly higher incidence of UTIs in the diabetic group. Females had an overall five-time higher risk of developing UTI in the diabetic group. There were no stark differences in the clinical and microbiological profiles of these patients; however, the diabetic group showed significantly more patients with asymptomatic UTI.

This study has provided substantial evidence to the comparatively higher risk of UTI in diabetic patients. However, it has its limitations too. This study was conducted in the OPD and only included clinically stable outpatient cases; hence, many cases with complicated UTI must have been missed. This study did not include the antibiotic sensitivity profile for both groups.

Previous studies reported the incidence of UTI in Pakistani diabetic patients to be $50 \%$ to $53 \%$ [9-11]. These figures are higher than those obtained in our study (13.7\%). In a Romanian study, the prevalence of UTIs in patients with DM was $12 \%$ [12]. An Indian study deduced the prevalence of asymptomatic bacteriuria to be significantly higher (28\%) among diabetic patients as compared to non-diabetics $(7.5 \% ; p=0.001)$ [13]. Higher incidence of UTI among females in the diabetic group as wells as in the general population has been reinforced in various studies [9-13]. In a study that compared the pattern of UTI in diabetic and non-diabetic females, it was seen that uncontrolled diabetes was associated with increasing severity of UTI. E. coli was the most commonly isolated pathogen in both groups. Candida was only seen in diabetic females group [14]. E. coli remained the most common pathogen in both groups of this study. Only $5 \%$ of cases of Candida were reported in the diabetes group. Pseudomonas was also only reported in the diabetes group in this study. Diabetics individuals are in a immunosuppressed state, hence at a greater risk of contracting Pseudomonas infection. Compared to the incidence of Pseudomonas in this study (14\%), other studies from Pakistan have reported varied incidence. Ijaz et al. reported that among diabetics, $72 \%$ urinary samples were positive for Pseudomonas; Bashir et al. reported that 1\% cases of Pseudomonas were isolated, and Zahra et al. $6 \%$ cases of Pseudomonas were isolated from non-diabetic urinary samples and none from the diabetic population [9-11]. In non-diabetic Pakistanis, 5\% urinary samples were found to be positive for Pseudomonas [15]. In an Indian diabetic sample of 651 culture-positive UTI, the frequency of Pseudomonas was $2.7 \%$; similar to our study, $E$. coli was also the most commonly isolated pathogen (69\%) [16].

Asymptomatic pyuria was significantly more common in the diabetic group as compared to non-diabetic in this study ( $30 \%$ vs. $5.6 \% ; p=0.03$ ). In an Indian study, asymptomatic bacteriuria was found in $40 \%$ of urinary samples in a diabetic population. Hematuria was reported in $4 \%$ of their samples, as compared to $5.7 \%$ in our diabetic samples. The most common isolate in their study was also E. coli [17]. In a Sudanese diabetic sample, the frequency of UTI was $19.5 \%$. Asymptomatic bacteriuria was present in $21 \%$ of these patients [18]. Clinical presentation of UTI in both groups was comparable in our study. Similarly, no significant difference was seen in the clinical presentation in Aswani et al. [5]. Even the frequency of asymptomatic bacteriuria was similar (30\%) in both diabetic and non-diabetic groups in their analysis [5]. According to the Infectious Disease Society of America (IDSA) guidelines, diabetic patients should not be screened or treated for asymptomatic bacteriuria [19]. When clinical signs are present, UTIs are to be treated as per the culture and sensitivity report.

\section{Conclusions}

The frequency of UTIs is higher in the diabetic population as compared to their non-diabetic counterparts. 
UTIs are more common among females in both groups. Clinical presentation in the two groups is also similar. Asymptomatic bacteriuria is a more common entity in diabetic patients and does not require any treatment.

\section{Additional Information \\ Disclosures}

Human subjects: Consent was obtained by all participants in this study. Dow University of Health Sciences issued approval IRB-021/2016. Animal subjects: All authors have confirmed that this study did not involve animal subjects or tissue. Conflicts of interest: In compliance with the ICMJE uniform disclosure form, all authors declare the following: Payment/services info: All authors have declared that no financial support was received from any organization for the submitted work. Financial relationships: All authors have declared that they have no financial relationships at present or within the previous three years with any organizations that might have an interest in the submitted work. Other relationships: All authors have declared that there are no other relationships or activities that could appear to have influenced the submitted work.

\section{References}

1. Abu-Ashour W, Twells L, Valcour J, Randell A, Donnan J, Howse P, Gamble JM: The association between diabetes mellitus and incident infections: a systematic review and meta-analysis of observational studies. BMJ Open Diabetes Res Care. 2017, 5:000336. 10.1136/bmjdrc-2016-000336

2. Sahib AKY: Study of ciprofloxacin resistant Escherichia Coli (CREC) in type 2 diabetic patients with symptomatic urinary tract infection. Iraq J comm Med. 2008, 21:58-63.

3. Baqai R, Aziz M, Rasool G: Urinary tract infection in diabetic patients and biofilm formation of uropathogens. Infect Dis J Pak. 2008, 17:7-9.

4. Wittig L, Carlson KV, Andrews JM, Crump RT, Baverstock RJ: Diabetic bladder dysfunction: a review . Urology. 2019, 123:1-6. 10.1016/j.urology.2018.10.010

5. Aswani SM, Chandrashekar UK, Shivashankara KN, Pruthvi BC: Clinical profile of urinary tract infections in diabetics and non-diabetics. Australas Med J. 2014, 7:29-34.

6. Woldemariam HK, Geleta DA, Tulu KD, Aber NA, Legese MH, Fenta GM, Ali I: Common uropathogens and their antibiotic susceptibility pattern among diabetic patients. BMC Infect Dis. 2019, 19:43. 10.1186/s12879-018-3669-5

7. Pramod KJ, Ratna B, Basudha K: Prevalence of uropathogens in diabetic patients and their susceptibility pattern at a tertiary care center in Nepal-a retrospective study. Int J Biomed Lab Sci. 2014, 3:29-34.

8. Bollestad M, Vik I, Grude N, Blix HS, Brekke H, Lindbaek M: Bacteriology in uncomplicated urinary tract infections in Norwegian general practice from 2001-2015. Brit J Gen Pract. 2018, 1:10.3399/bjgpopen17X101145

9. Ijaz M, Ali SA, Khan SM, Hassan M, Bangash IH: Urinary tract infection in diabetic patients; causative bacteria and antibiotic sensitivity. J Med Sci. 2014, 22:110-114.

10. Bashir H, Saeed K, Jawad M: Causative agents of urinary tract infection in diabetic patients and their pattern of antibiotic susceptibility. Khyber Med Univ J. 2017, 9:201-204.

11. Zahra N, Rehman K, Aqeel R, Parveen A, Akash MSH: Assessment of urinary tract infection and their resistance to antibiotics in diabetic and non-diabetic patients. Bangabandhu Sheikh Mujib Med Univ J. 2016, 9:151-155.

12. Chiţă T, Timar B, Muntean D, et al.: Urinary tract infections in Romanian patients with diabetes: prevalence, etiology, and risk factors. Ther Clin Risk Manag. 2017, 13:1-7. 10.2147/TCRM.S123226

13. Hiamanshu D, Singhal S, Vaish AK, Singh M, Rana H, Agrawal A: A study of asymptomatic bacteriuria in North Indian type 2 diabetic patients. Int J Diabetes Dev Ctries. 2017, 37:42-45. 10.1007/s13410-015-0430-0

14. Garg V, Bose A, Jindal J, Goyal A: Comparison of clinical presentation and risk factors in diabetic and nondiabetic females with urinary tract infection assessed as per the european association of urology classification. J Clin Diagn Res. 2015, 9:14. 10.7860/JCDR/2015/14177.6029

15. Shah DA, Wasim S, Abdullah FE: Antibiotic resistance pattern of Pseudomonas aeruginosa isolated from urine samples of urinary tract infections patients in Karachi, Pakistan. Pak J Med Sci. 2015, 31:341-345. 10.12669/pjms.312.6839

16. Jagadeeswaran G, Ansari MZ, Rajangam T: Urinary tract infection in diabetics - a five year retrospective study on the prevalence of bacterial isolates and its antibiotic susceptibility patterns in a tertiary care hospital in South India. Int J Contemp Med Res. 2018, 5:33-38. 10.21276/ijcmr.2018.5.4.45

17. Nongrum S, Thaledi S, Singh VA, et al.: Association of uropathogens with asymptomatic urinary tract infection in diabetes mellitus patients. Int J Curr Microbiol App Sci. 2016, 5:355-361. 10.20546/ijcmas.2016.510.040

18. Hamdan HZ, Kubbara E, Adam AM, Hassan OS, Suliman SO, Adam I: Urinary tract infections and antimicrobial sensitivity among diabetic patients at Khartoum, Sudan. Ann Clin Microbiol Antimicrob. 2015, 14:26. 10.1186/s12941-015-0082-4

19. Nicolle LE, Gupta K, Bradley SF, et al.: Clinical practice guideline for the management of asymptomatic bacteriuria: 2019 update by the Infectious Diseases Society of America. Clin Infect Dis. 2019, 68:83-110. $10.1093 /$ cid/ciy1121 